Portland State University

PDXScholar

\title{
Solving Chemical Problems of Environmental Importance Using Cavity Ring-Down Spectroscopy
}

\author{
Dean B. Atkinson \\ Portland State University, atkinsond@pdx.edu
}

Follow this and additional works at: https://pdxscholar.library.pdx.edu/chem_fac

Part of the Chemistry Commons

Let us know how access to this document benefits you.

\section{Citation Details}

Atkinson, D. B. (2003). Solving chemical problems of environmental importance using cavity ring-down spectroscopy. The Analyst, 128(2), 117-125.

This Article is brought to you for free and open access. It has been accepted for inclusion in Chemistry Faculty Publications and Presentations by an authorized administrator of PDXScholar. Please contact us if we can make this document more accessible: pdxscholar@pdx.edu. 


\title{
Solving chemical problems of environmental importance using cavity ring-down spectroscopy
}

\author{
Dean B. Atkinson* \\ Department of Chemistry, Portland State University, Portland, OR 97207-0751, USA
}

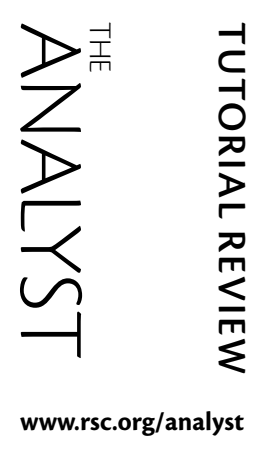

Received 10th July 2002, Accepted 7th November 2002

First published as an Advance Article on the web 5th December 2002

Cavity ring-down (CRD) is a sensitive variant of traditional absorption spectroscopy that has found increasing use in a number of chemical measurement applications. This review focuses on applications of cavity ring-down spectroscopy that will be of interest to environmental chemists and analytical chemists working on environmental problems. The applications are classified into direct monitoring approaches, indirect analysis methods and ancillary studies and a differentiation is made between field-tested instruments and proof of principle studies.

\section{Background}

Shortly after the introduction of cavity ring-down (CRD) spectroscopy, one of its progenitors predicted that a large number of environmental measurement problems would be addressed by the technology ${ }^{1}$ and indeed that statement is beginning to come to fruition. In this review there will only be a cursory explanation of the two manifestations of the technique which are currently referred to as ring-down spectroscopy since a large number of comprehensive review articles and books have already been published on the details of the technique. ${ }^{2-5}$ Because of length considerations, this review will also not treat experiments with the related techniques referred to as cavity enhanced absorption and integrated cavity output spectroscopy (CEAS and ICOS $)^{6-8}$ or the extremely sensitive but technically challenging noise immune cavity enhanced optical heterodyne molecular spectroscopy (NICE-OHMS). ${ }^{9}$ No review of an evolving topic can claim to be complete, but some effort has been made here to at least be representative of the types of studies which will be of interest to environmental chemists and those groups world-wide who have been involved in them. Accidental omission of important work is regrettably quite likely because of the breadth of the extant literature and as a

Dean B. Atkinson received his $P h D$ at the University of Arizona in 1995. He was a postdoctoral fellow at NIST in Gaithersburg, MD from 1995-1997 and in 1997 accepted an Analytical Chemistry faculty position in the Chemistry Department at Portland State University. His research specializes in the

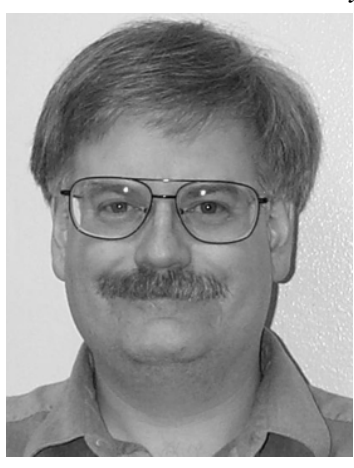
development and implementation of powerful new measurement methodologies to chemical problems of environmental importance. Projects ongoing in his laboratory include the measurement of chemical reaction rates of organic peroxy radicals of importance in the terrestrial atmosphere and the determination of ambient aerosol optical properties using cavity ring-down spectroscopy. recent CRD reviewer notes, "more and more papers appear with titles without the words "cavity ring-down."”2

Where specific details of the experimental approach are known to limit or enhance the application to environmental problems, these details will be identified, but the reader will be directed to other sources for a fuller discussion. This review will classify applications into three subject classes: direct monitoring approaches, indirect analysis methods, and ancillary studies. Within the direct monitoring subject, which represents the largest number of studies with the most obvious impact on environmental chemistry, the review will also differentiate between field-tested instruments and proof-of-principle studies. In a short conclusion, a prediction of the future applications of CRD systems to environmental problems will be attempted.

\section{An introduction to cavity ring-down spectroscopy}

This section is mainly a qualitative description, meant to give an understanding of the technique for the uninitiated. Other essentially equivalent descriptions are given in the cited literature. ${ }^{10-13}$ Cavity ring-down spectroscopy is based on the difference between measurements of the rate at which light intensity leaks out of a stable optical cavity (see Fig. 1) with and without the analyte present. This measurement can then be easily converted to optical extinction, scattering coefficient,

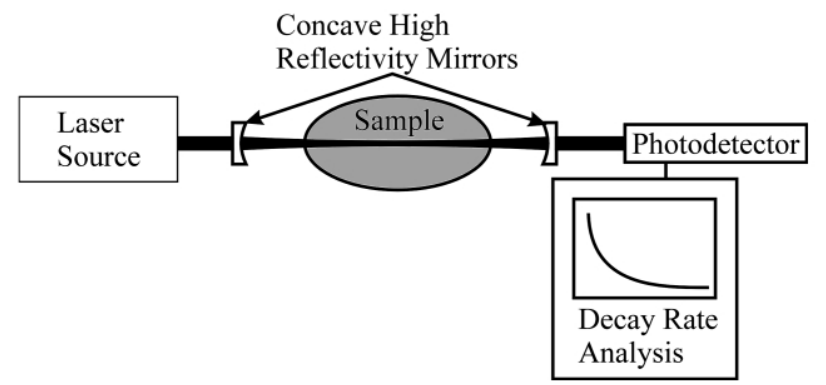

Fig. 1 An experimental diagram showing the essential features of any cavity ring-down experiment. Most experiments use a digital oscilloscope and computer to extract the decay constant. Many also include pre-cavity optics (e.g., spatial filters, mode-matching lenses, and for most CW-CRD experiments, acousto-optic modulators and opto-isolators). The most common type of CW-CRD set-up includes cavity length modulation, typically by a piezo-transducer attached to one end of the cavity. 
absorbance, or other commonly encountered analytical quantities as described below.

The cavity is usually formed by two or more highly reflective mirrors, at least one of which is concave to induce stability. ${ }^{14}$ The reflectors for these experiments are typically fashioned from highly polished transmissive substrates (often quartz) with special multi-layer dielectric coatings. Reflectivities of $99.999 \%$ or greater have been reported. During the experiment a finite amount of light is injected into the cavity, bounces back and forth while becoming less intense due to the various loss processes offered by the cavity and sample, and is monitored by an appropriately placed detector outside the cavity. (This is the origin of the term 'ring-down', the photons are visualized as ringing back and forth in the cavity while decaying down in time-visualize a bell ringing after having been struck.) A key assumption is that the fraction of light detected at any time is uncorrelated with intensity or its time history and thus the detector signal mimics the intensity inside the cavity. The timedependent photo-induced electrical signal from the detector is analyzed by one of a number of methods. ${ }^{15-17}$ The most popular way is to digitize the signal and extract the decay constant $\beta$ from the digital data

$$
S(t)=S_{0} \exp (-\beta t)+C
$$

where $S$ and $S_{0}$ are the observed signal and the signal at the beginning of the fit, and $C$ is the signal offset level. The units of $S$ and $C$ are irrelevant; they can be photon flux, signal voltage, or simply the digital representation of a voltage, typically an integer. The magnitude of $S_{0}$ is also unimportant, which is why the technique is useful with pulsed lasers which typically fluctuate in intensity by 5 to $10 \%$ per pulse. Often the inverse of the decay constant, or ring-down time $\left(\tau=\beta^{-1}\right)$ is cited, resulting in slightly different expressions than those given here.

The derived value of $\beta$ is the rate constant for the first order loss of light intensity from the cavity. When the value of $\beta$ for the blank (no analyte present) is subtracted from that obtained with the analyte present $\left(\beta_{\text {sample }}-\beta_{\text {blank }}\right)$ the result is the corresponding rate constant for light intensity loss due only to the analyte, which is the optical extinction in the uncommon units of inverse time $\left(\mathrm{s}^{-1}\right)$. Since the speed of light is very nearly a constant during a given experiment $\dagger$ a simple conversion

$$
\text { Ext. }\left(\mathrm{m}^{-1}\right)=\left(\beta_{\text {sample }}-\beta_{\text {blank }}\right)\left(\mathrm{s}^{-1}\right) / \text { speed of light }\left(\mathrm{m} \mathrm{s}^{-1}\right)
$$

gives the extinction coefficient in the more commonly used units of inverse length $\left(\mathrm{m}^{-1}\right.$ or $\left.\mathrm{cm}^{-1}\right)$. It is sometimes desirable to multiply the extinction coefficient by the length of the cavity to obtain the unitless extinction per pass through the cavity,

$$
\ln \left(I_{0} / I\right)=\text { Ext. }\left(\mathrm{m}^{-1}\right) \times \text { cavity length }(\mathrm{m})
$$

to obtain the quantity, i.e., $\ln \left(I_{0} / I\right)$, analogous to that typically measured in absorption or extinction instruments. If the extinction is small, its value is nearly equal to the fractional light intensity loss (typically given in parts-per-million, $\mathrm{ppm}$ ) in the same units (per time, per length, or unitless), because $\ln (1-$ $x)^{-1} \sim x$ for small $x$.

The interpretation of the extinction derived from cavity ringdown measurements depends on the experiment being performed. In most of the studies reported to date, the dominant source of extinction was absorption; but CRD has also been used to probe total extinction, ${ }^{18}$ scattering by aerosol particles, ${ }^{19}$ and rotation of plane polarized light, ${ }^{20}$ and there are other extinction-related properties which can be investigated. For systems where the absorption of light is of interest, it is

$\dagger$ The speed of light will not be constant in all experimentally encountered situations, however. For example, gas phase and solution phase measurements are taken in sample matrices with significantly different refractive indices and hence speeds of light. convenient to transform the quantity in eqn. (3) to the common $\log$ version to obtain the defined ${ }^{21}$ quantity absorbance.

$$
\text { Absorbance }=\log \left(I_{0} / I\right)=\ln \left(I_{0} / I\right) /(\ln (10) \sim 2.303)
$$

The absorbance may then be used in the normal Beer's law sense to determine concentration from known absorption strength or vice versa

Absorbance $=$ concentration $(c) \times$ absorption strength $(\varepsilon) \times$ pathlength $(l)$

where the absorption pathlength may be equal to the cavity length or not, depending on the experimental design. (As usual, some care should be taken when using published values of the absorption strength, which are sometimes base $e$ and sometimes base 10).

When using the measured extinction directly, or interpreting it as absorption, scattering or some other extinction-related quantity, it is important to realize that the pathlength of the interaction may not equal the cavity length, necessitating a treatment like that in eqns. (3) and (5) above. Also, the careful choice of a blank system (rather than the commonly used evacuated cavity) for the difference measurement in eqn. (2) can enhance the utility of a CRD measurement. Some examples are: (1) in spectroscopic measurements, the off-resonance absorption has been used many times as the 'blank', ${ }^{16,17}$ (2) for measurements of the particle-induced extinction of aerosols, we use clean air produced by filtering the sample, ${ }^{19}$ (3) for kinetics measurements of radicals produced by laser photolysis, the prephotolysis sample may be used as a blank. ${ }^{22}$ In all of these cases, extinction due to the sample matrix is removed in addition to the effects of the mirror-related loss mechanisms (scattering, absorption, and transmission by the mirrors).

The sensitivity of the CRD extinction measurement [eqn. (2)] is related to the size of the lowest cavity loss obtainable, ${ }^{10}$ and is typically of the order of $1 \times 10^{-6}(\mathrm{ppm})$ per pass through the cavity. This represents a considerable improvement over most simple absorption and extinction measurements. The improvement is presumably due to the combined effects of increased pathlength (several kilometers can be produced with good mirrors), over sampling of the intensity change (typically a point is digitized every few nanoseconds), and the overall insensitivity to fluctuations in the source intensity and detectivity. The extinction measurements have been demonstrated to be randomly distributed, so signal averaging results in the usual $\mathrm{N}^{-0.5}$ decrease in the standard deviation of the mean. ${ }^{23}$ (Careful investigators in CRD are beginning to provide sensitivity specifications that include the $\mathrm{Hz}^{-0.5}$ unit, rather than citing a particular integration time.)

\section{Variants of the original pulsed technique}

To this point, no mention has been made of the source of the light or how it is injected into the cavity. While the original technique used pulsed lasers to produce the light, a variant has emerged which uses continuous lasers. The two techniques will be referred to in this review as pulsed CRD and CW-CRD. (Some authors apparently felt that since the CRD technique inherently involves a changing light level, the use of CW was confusing, so they call the technique cavity leak-out spectroscopy or CALOS.) ${ }^{24}$ When pulsed lasers (usually with nanosecond pulse widths) are used to excite the cavity, the intensity at the photodetector (and by inference in the cavity) is observed to rapidly peak at a high value and decay as expected after that. When continuous lasers are used, the situation is usually different. First, the instantaneous power of continuous lasers is lower than that of pulsed lasers and is often concentrated in a narrow bandwidth. The cavity presents a set of very narrow transmission fringes owing to its high finesse, ${ }^{25}$ which must be 
matched to the laser bandwidth to allow light injection. Most investigators have chosen to match the laser's output spectrum to the cavity's transmission spectrum either by modulating the cavity length ${ }^{26}$ or the laser ${ }^{27}$ or both. Alternatively, a large number of transverse modes of the cavity can be used to "fill in" the frequency spectrum and higher laser power can be used to ascertain that some gets through. ${ }^{28,29}$ Second, when light appears at the detector and signal is observed, this implies that light intensity exists in the cavity, and the light influx must be turned off to monitor the decay (ring-down). This has been accomplished in a number of ways, but most often by using an acousto-optic modulator. ${ }^{26,29,30}$

The CW-CRD technique is more involved than the pulsed variant, so why is it used? The answer lies in the low cost and high performance of tunable diode lasers. These devices are typically run continuously with output energies in the $\mathrm{mW}$ range with good wall-plug efficiency (normal line voltages of $110 / 230$ VAC) and are readily available through the nearinfrared (NIR) and visible. Continuous lasers, be they diodes or otherwise, can provide much narrower bandwidths (typically less than $1 \mathrm{MHz}$ ) than can be obtained with pulsed lasers. This is fortunate in CW-CRD, since the matching of the laser to the cavity transmissions is only effective if they are of comparable width, but it also implies that very high resolution can be obtained in spectroscopy. Another advantage is duty cycleCW-CRD setups can achieve measurement rates of 10 's of $\mathrm{Hz}$ to $\mathrm{kHz} .{ }^{27,31}$ Finally, the matching between the laser and a single transverse and longitudinal cavity mode produces more consistent ring-downs because of more constant diffraction losses, resulting in some of the best sensitivities yet reported for CRD. ${ }^{31}$ These points were clear to K. K. Lehmann who filed patent almost immediately after the demonstration of the CWCRD technique. ${ }^{32}$

A particularly promising innovation on the CRD approach is the multi-wavelength ring-down measurement, originally called ringdown spectral photography RSP. ${ }^{33}$ Although this technique is still undergoing development, ${ }^{34,35}$ one group has already demonstrated its use in direct atmospheric monitoring. ${ }^{36}$ The method uses two-dimensional array detectors to monitor the light intensity exiting a cavity as it decays in time and is dispersed in wavelength. A compelling illustration of the concept is provided in Fig. 2 where a single wavelength laser was detected in the time and wavelength sensitive manner as it was step-scanned across the operating range and the time averaged image is shown. ${ }^{33}$ Of course, in the final version of the method all wavelengths are incident on the cavity at once and the full time and wavelength dispersed image is recorded in a single ring-down event (see cover illustration, this issue). ${ }^{34}$ This method holds great promise for the measurement of species with broadened but distinct absorptions, such as are commonly encountered in the UV and visible, in complex sample matrices.

\section{Relative strengths and weaknesses of CRD in environmental applications}

Whether the application uses CW-CRD or pulsed CRD, single wavelength or broadband measurement, the advantages are similar: a high sensitivity optical extinction measurement is possible at low to moderate cost with low to moderate complexity. All share a similar downside as well; they are most effective in gas-phase, low base-loss configurations. This usually means that windows may not be used within the cavity, and whatever medium is present must be fairly transparent. In cases where the sample could affect the mirror reflectivity (e.g., by reaction or particle deposition) some care must be taken to protect the mirrors. ${ }^{22}$ They are also line of sight measurements, which has implications in sampling. ${ }^{37}$ Finally, the geometry of cavity plus detector and source can be somewhat restrictive in experimental design.

Since the fundamental measurement in CRD is optical extinction, it shares some of the strengths and weaknesses of other extinction or absorption-based spectroscopic methods used in environmental analytical chemistry (e.g., photo-acoustic spectroscopy PA, ${ }^{38}$ long-path absorption, and the related differential optical absorption spectrometry DOAS technique). ${ }^{39}$ Although spectral features of analytes may be sharp and distinct at low pressures, they become significantly broadened at atmospheric pressure (and even more so in solution). This impacts both the sensitivity (peak absorptivities decrease) and selectivity (broad features due to the analyte and interferences are harder to distinguish from one another) of measurements of analytes. As a case in point, the detection of $10^{13}$ molecules $\mathrm{cm}^{-3}$ of nitrogen dioxide at 1 torr total pressure of nitrogen reported in the next section will probably not extrapolate directly to a detection limit in ppm of atmospheric air because of broadening at the high pressure and/or analyte loss in getting samples to lower pressures, as well as the possibility that other compounds in air will absorb significantly at the monitor wavelength. The problem of spectral interferences is not restricted to absorbers for those methods that actually measure extinction-i.e., all but PA above-scattering by gases and particles can contribute to the observed signal as well.

At the other extreme of spectral feature width (the narrow lines which are observed in atomic absorption) all of the absorption/extinction techniques need to consider the effects of relative bandwidth and saturation. The issue of source bandwidth overlap with analyte linewidth is well known in atomic spectroscopy and is described in many analytical texts. ${ }^{40}$ If the requirement of a narrow source bandwidth with respect to the

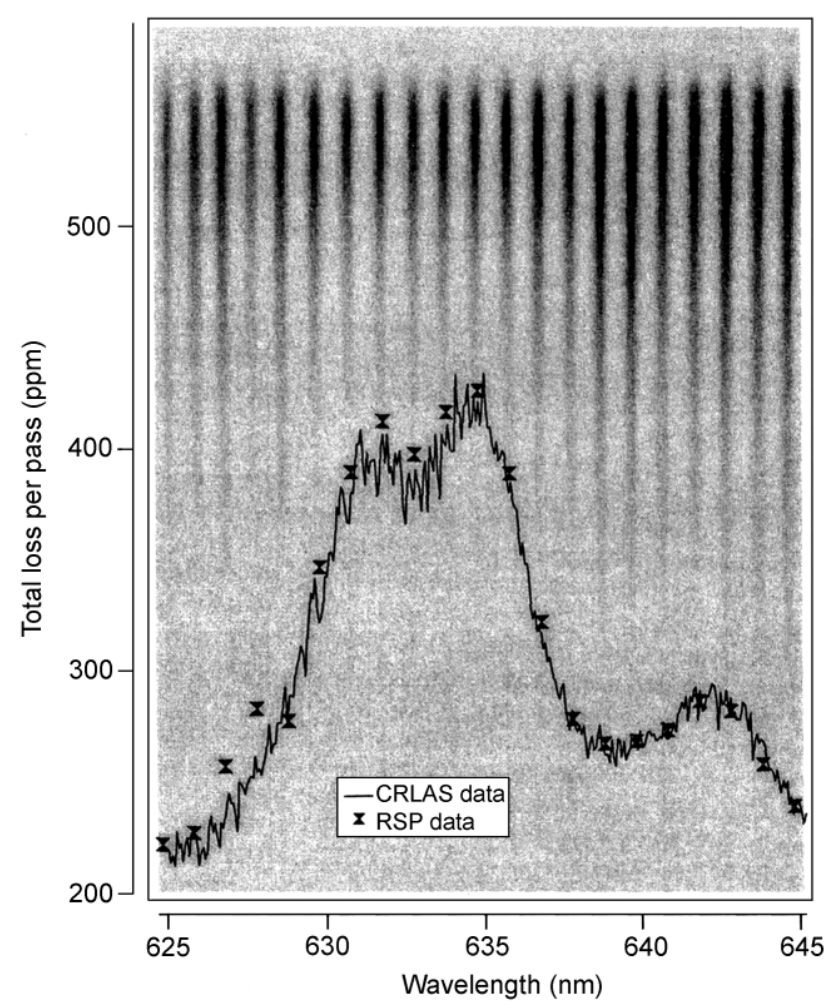

Fig. 2 A point-wise multi-wavelength ring-down experiment (ringdown spectral photography) is illustrated via the hourglass symbols and streak image. Individual single wavelength decays were dispersed in wavelength (the $x$ axis) and co-added to produce the streak image. Essentially the longer the streak continues, the lower the absorbance is at that wavelength. A normal pulsed CRD spectrum of the absorber propane is also shown for reference. Reprinted from Chem. Phys. Lett., 1998, 292, pp. 143-153, J. J. Scherer, 'Ringdown spectral photography'. Copyright (1998), with permission from Elsevier Science. 
atomic linewidth is fulfilled, the absorption measurements are quantitative, sensitive, and selective, if saturation effects can be avoided. Saturation is more likely in CW-CRD than in pulsed, because of the higher intra-cavity power that may be achieved on-resonance.

An advantage that CRD has over the methods that measure transmission through a long pathlength of ambient air is the ability to control the sample. For example, it is relatively straightforward to place an inlet filter on a CRD (or PA) experiment to remove aerosol particles either size selectively (impactors, vortex) or essentially completely (HEPA filter) resulting in an elimination (or measurement) of their contribution. Chemical species may also be removed (driers or denuders) but in all of these sample pretreatment methods, verification that the analyte concentration is not affected (or is properly calibrated) becomes important. Other advantages over long-path measurements include experimental concerns like better portability and more robust systems.

The power of DOAS, which is traditionally applied to longpath extinction measurements, lies in the data treatment, ${ }^{39}$ so it should not be regarded as a competitive method. Spectra obtained by CRD methods, especially the simultaneous multiwavelength variants, can be treated by the DOAS formalism to obtain the improved detection limits and freedom from interferences that it offers. The addition of the ability to pretreat the sample described above could considerably improve the usefulness of the DOAS/CRD marriage.

Cavity ring-down and photo-acoustic methods offer comparable absorbance/extinction sensitivities in the $10^{-10} \mathrm{~cm}^{-1}$ to $10^{-8} \mathrm{~cm}^{-1}$ range for typical experimental setups. ${ }^{3}$ Both can be considered absolute measurements. ${ }^{38,41}$ Some advantages that CRD may have over PA are experimental simplicity, cost, and stability, but no conclusive head-to-head comparisons have been made. The two methods are complementary in an important respect: in PA, only absorption is measured, while in CRD extinction is measured. In some atmospheric science problems, the ability to measure both may be more powerful than either alone.

The figures of merit (sensitivities, detection limits, dynamic ranges, etc.) which are quoted in this review are those cited in the original papers, although in some cases, comments are made about the method of their determination. Mixing ratios are often cited in ppmv, ppbv, and pptv (parts per million, billion and trillion by volume, respectively in atmospheric pressure air) extrapolated from low pressure, high mixing ratio measurements, so some care should be taken in establishing what is actually measured. In any environmental monitoring application, the best estimates of the eventual success of an approach are obtained in measurements of the target analyte in a representative sample matrix, where the effects of interfering species can be properly assessed. Comparison with existing measurement methodology is also very valuable in validating the accuracy and applicability of new methods.

\section{Subject 1-direct monitoring of environmental contaminants}

Although a large number of species have been detected in the gas phase by $\mathrm{CRD}^{2}$ those that appear to be likely candidates for direct atmospheric detection fall into four classes: (1) nitrogen oxides and HONO, (2) elemental mercury and volatile mercury compounds, (3) hydrocarbons, and (4) particulate matter in the atmospheric aerosol. Of these, all but hydrocarbons have been measured by CRD in the nascent atmosphere and portable field instruments have been reported for $\mathrm{NO}_{3}$ and $\mathrm{N}_{2} \mathrm{O}_{5}{ }^{42}$ and for aerosols. ${ }^{19}$

\section{Nitrogen oxides and HONO}

\section{Nitrogen dioxide}

The oxides of nitrogen are deeply involved in atmospheric chemistry and photochemistry and ambient measurements of $\mathrm{NO}_{2}$ and $\mathrm{NO}$ (collectively referred to as $\mathrm{NO}_{x}$ ) are routinely performed in most urban areas, as are automobile exhaust and stack monitoring. ${ }^{43}$ Ambient atmospheric measurement of $\mathrm{NO}_{2}$ is still an active research topic, despite the availability of commercial instruments and its readily accessible spectral features. The detection/measurement of $\mathrm{NO}_{2}$ by CRD has been reported many times with detection limits from the high ppmv ${ }^{44}$ to $100 \mathrm{ppbv}$ range, ${ }^{45}$ but all are laboratory measurements in a controlled atmosphere. ${ }^{1,44-49}$ (In fact, two of these studies were actually recordings of the spectrum of jet-cooled $\mathrm{NO}_{2}$. $)^{46,47}$

Nitrogen dioxide is an interesting analyte: in a sense it is ideally suited to detection by optical spectroscopy, having absorptions that extend essentially continuously from the UV through the visible and a natural fluorescence quantum yield near unity. Unfortunately, strong quenching by air limits fluorescence measurements of $\mathrm{NO}_{2}$ to considerably reduced pressure (decreasing sensitivity and increasing experimental complexity) and the feature density of the absorption spectrum makes conventional differential spectral measurement (i.e., on resonance vs. off) difficult. The absorption features of $\mathrm{NO}_{2}$ are also strongest near $450 \mathrm{~nm}$ where a larger number of possible interfering compounds absorb. The best prospects for future success in ambient measurement by CRD will likely be attained by concentrating on the visible bands, where only overtones of water vapor and aerosol scattering (which can be removed) are likely to be serious interferences, and by applying either a multispectral measurement ${ }^{34,36}$ or at least a two-wavelength difference measurement in the calibration. For both of the latter approaches, the base sensitivity of the method is only indirectly related to the smallest observable change in ring-down decay rate, so experiments will be needed to provide reliable estimates of the detection limits and other figures of merit.

\section{Nitric oxide}

Ambient measurement of NO by commercially available instrumentation is facile and reliable but is sometimes unsuitable for measurements in difficult matrices like human breath or engine exhaust. A recent report described a pulsed CRD instrument which measures $\mathrm{NO}$ (at $212 \pm 22 \mathrm{ppm}$ ) and $\mathrm{NO}_{2}$ (at $29 \pm 4 \mathrm{ppm}$ ) simultaneously (at different wavelengths) in diesel exhaust. ${ }^{44}$ Although these are not impressive detection limits for either species, they are suitable for the intended application and the simultaneous sensing is an interesting approach. An impressive detection limit of $0.7 \mathrm{ppbv}$ is reported by monitoring NO in the mid-infrared at $5.2 \mu \mathrm{m}$ using a CW-CRD method based on a quantum-cascade distributed-feedback laser. ${ }^{50}$ Unfortunately, the wavelength used in these experiments was shown to be strongly interfered with by nearby lines of $\mathrm{CO}_{2}$, which would complicate its application in the atmosphere and in human breath (for which the experiment was attempted).

\section{Nitrogen trioxide and dinitrogen pentoxide}

The nitrate radical $\mathrm{NO}_{3}$ has been suggested to be an important night-time (it is rapidly photolyzed by daylight) atmospheric oxidant. ${ }^{39,43} \mathrm{NO}_{3}$ has been measured in the ambient atmosphere, but improved detection methods (lower detection limitdown to sub-pptv, in situ, more portable, etc.) are needed. The reservoir species $\mathrm{N}_{2} \mathrm{O}_{5}$, which is in equilibrium with separated $\mathrm{NO}_{3}$ and $\mathrm{NO}_{2}$ and is thus an important component in the nitrogen oxide cycle, has not been directly detected in the 
atmosphere. Three groups have reported measurements of $\mathrm{NO}_{3}$ using CRD ${ }^{36,42,51}$ and are working on prototypes of CRD field instruments, one of which has been deployed. ${ }^{42}$ The absorption (in the visible near $660 \mathrm{~nm}$ ) used in all of the optical measurements is a nearly ideal case in terms of lack of spectral interference. Only atmospheric aerosol particle induced scattering (which may be mitigated by simple filtering) is found to be a significant problem and a detection limit of $0.3 \mathrm{pptv}$ (parts per trillion by volume) has been reported in the pulsed CRD fieldoperable instrument. ${ }^{42}$

A non-trivial point which is highlighted in Brown et al. is the careful handling of 'real' air samples to minimize refractive index gradients and other potential problems with the sensitive measurement of the optical extinction in ambient measurements. ${ }^{42}$ This instrument also allows the measurement of the sum of $\mathrm{NO}_{3}+\mathrm{N}_{2} \mathrm{O}_{5}$ through online conversion of $\mathrm{N}_{2} \mathrm{O}_{5}$ in a heated inlet system to $\mathrm{NO}_{3}$, an inspired extension of the usefulness of the instrument. Typical results for the system are shown in Fig. 3, along with simultaneous measurements of ozone and the air temperature. Clearly the system is sufficiently sensitive to easily measure both nitrogen oxides in the nascent atmosphere and current work will allow both $\mathrm{NO}_{3}$ and the sum $\mathrm{NO}_{3}+\mathrm{N}_{2} \mathrm{O}_{5}$ (and hence $\mathrm{N}_{2} \mathrm{O}_{5}$ alone) to be measured simultaneously. 52

One of the other reported $\mathrm{NO}_{3} \mathrm{CRD}$ experiments uses the multi-wavelength approach. ${ }^{36}$ This proof-of-principle instrument only attained a sensitivity of a 'few pptv', but experimental improvements are expected and the detection of compounds via a range of wavelengths has proven to be a very robust measurement strategy as noted above. The third $\mathrm{NO}_{3}$ instrument uses the CW-CRD method ${ }^{51}$ and also reported higher detection limits of 2 pptv (noise-equivalent-absorption) which would likely need to be revised upward. This final strategy has the greatest opportunity for miniaturization and portable deployment of the three, since it is based on a tunable diode laser and all solid state components. If all three instruments ${ }^{36,42,51}$ are shown to provide comparable quality measurements there may be an interesting synergy in their application.

\section{Nitrous acid}

Nitric acid and $\mathrm{HONO}$, the products of $\mathrm{OH}$ radical reaction with $\mathrm{NO}_{x}$, are important reservoirs for both reactants. ${ }^{39}$ Their measurement (especially HONO) in the ambient atmosphere is of interest. Quantitation of HONO has been reported at levels down to $5 \mathrm{ppbv}$ with an impressive dynamic range up to 10 ppmv using both strong and weak absorption features. ${ }^{53} \mathrm{~A}$ pulsed CRD system was used to access a number of UV transitions near $354 \mathrm{~nm}$ (also used in the conventional DOAS measurements). ${ }^{54}$ The incorporation of two-wavelength differential absorption should help further reduce the effects of interferences and in combination with experimental improvements, result in lower detection limits.

\section{Mercury and its compounds}

The detection of elemental mercury vapor in laboratory air and its measurement in simulated ambient air by CRD have been reported. ${ }^{55,56}$ Mercury is unique, possessing a volatile atomic elemental form, and is highly toxic. As such, its detection via the narrow cluster of strong atomic absorptions (due to the different isotopic forms and fine structure transitions) near $253.6 \mathrm{~nm}$ represents another near-ideal case (if saturation effects are properly considered) in absorption measurement of environmental importance. A detection limit of $0.5 \mathrm{pptv}$ in air and a dynamic range to $50 \mathrm{pptv}$ is reported and the interferences presented by ozone and $\mathrm{SO}_{2}$ were examined. ${ }^{56}$ Detection of $\mathrm{HgCl}_{2}$ was also reported in the same setup since the spectrum of mercury compounds resembles that of the atom. The authors noted that speciation may require auxiliary sample pretreatment.

\section{Volatile hydrocarbons}

The ambient measurement of organic compounds emitted by natural and man-made sources is an important tool which aids in our understanding of the chemistry of the atmosphere. ${ }^{43}$ Methane is particularly important in global climate change, since it is the second largest anthropogenically produced direct chemical source of greenhouse warming after $\mathrm{CO}_{2}$. Isotopic ratio measurements of ${ }^{13} \mathrm{CH}_{4} /{ }^{12} \mathrm{CH}_{4}$ may be used to help determine the sources and sinks of methane in the environment. Gas chromatographic methods for the measurement of ambient mixing ratios and the isotopic ratio of methane (and other hydrocarbons) are robust and versatile, but the time per analysis is long, so some effort has gone into developing more real-time and portable systems for specialized applications like plume tracking. Cavity ring-down (and variants like CEAS) ${ }^{57,58}$ has begun to be explored for direct real-time spectroscopic measurement of hydrocarbons.

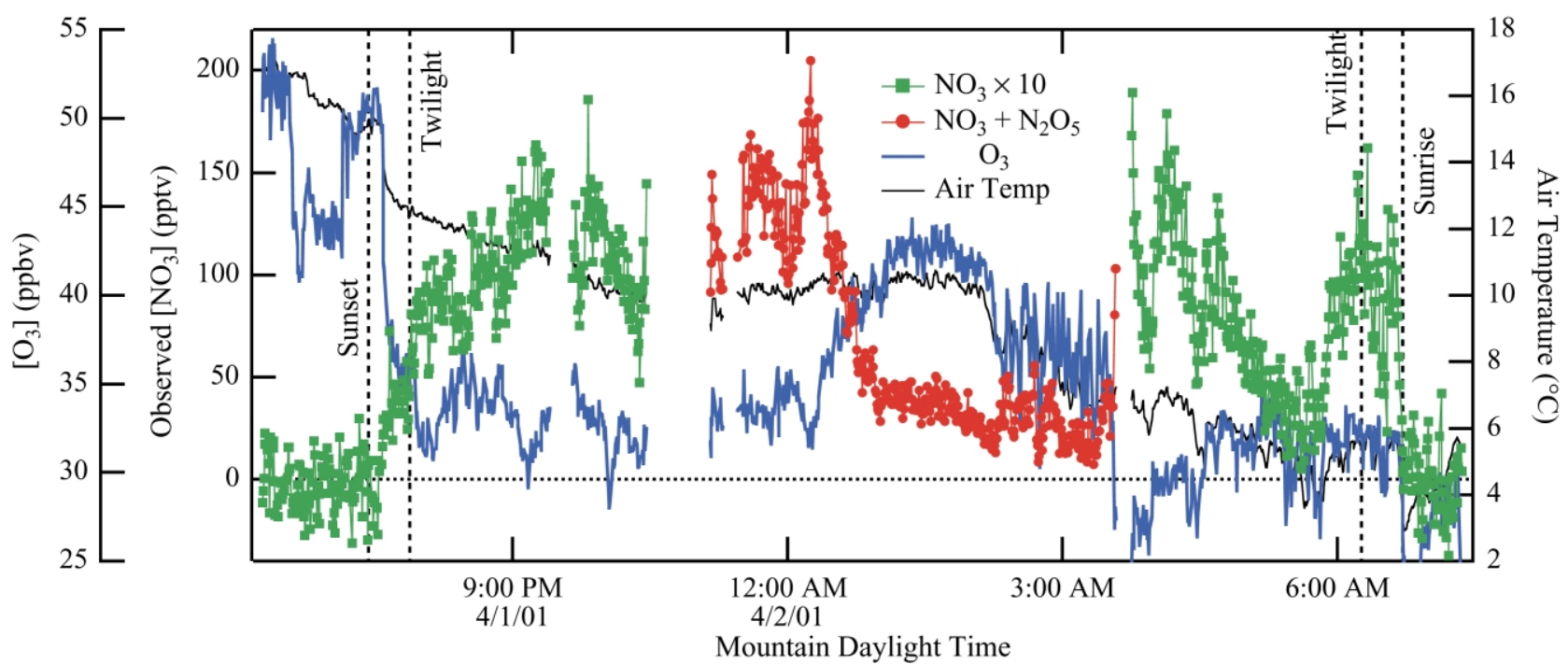

Fig. 3 Alternately measured mole fractions of $\mathrm{NO}_{3}$ (ambient temperature inlet, green squares) and the sum of $\mathrm{N}_{2} \mathrm{O}_{5}+\mathrm{NO}_{3}($ heated inlet, red circles), along with ozone (blue line, second left axis) and sampled air temperature (black line, right axis) on the night of April 1-2, 2001. The $\mathrm{NO}_{3}$ mole fractions have been multiplied by 10 to make them more visible on the scale of the $\mathrm{N}_{2} \mathrm{O}_{5}+\mathrm{NO}_{3}$ data. Reproduced by permission of American Geophysical Union. 
Hydrocarbons (especially alkanes) are transparent in the visible and near-UV regions of the spectrum where the best combinations of tunable sources, high quality mirrors, and highly sensitive detectors for cavity ring-down are usually found. The best opportunity for sensitive and general detection of hydrocarbons is in the mid-infrared 'fingerprint' region of the spectrum. (Actually this statement is fundamentally true for all molecular analytes, but as the density of accessible species increases, so does the probability of spectral interference.) The greatest limitation to performing CRD experiments in the midIR has been the difficulty of obtaining sufficiently intense narrow bandwidth tunable laser sources, and the development of CRD measurements has only slightly lagged behind the introduction of the novel sources (e.g., the $\mathrm{CO}$ overtone side band lasers ${ }^{59}$ and pulsed PPLN OPG/OPA systems described later). 60

CW-CRD measurements of methane and ethylene were reported in the mid-IR using tunable $\mathrm{CO}$ overtone sideband radiation near $3 \mu \mathrm{m}$ and tunable narrowband $\mathrm{CO}_{2}$ radiation near $10 \mu \mathrm{m} .24,59,61$ The authors have chosen to refer to the technique as cavity leak-out spectroscopy or CALOS, but their implementation is very similar to other methods which use continuous excitation (CW-CRD). The detection limit of the method is cited as being at the 'ppbv level' for ethylene and methane at $3 \mu \mathrm{m}$ and $1 \mathrm{ppbv}$ for ethylene at $10 \mu \mathrm{m}$, although all of the experiments to date appear to have been conducted on dilute mixtures in nitrogen. The identities and impact of potential interferences at these wavelengths were not discussed. The determination of the isotopic composition $\left(\delta^{13} \mathrm{C}\right)$ of methane in natural air was also reported using the $\mathrm{CO}$ laser system near $3 \mu \mathrm{m} .{ }^{62}$

A promising system for the measurement of atmospheric hydrocarbons and other pollutants in the mid-IR is based on a new type of source, optical parametric generation and amplification (OPG/OPA) by periodically poled lithium niobate (PPLN). ${ }^{60}$ The detection of methane via the structured absorptions near $3020 \mathrm{~cm}^{-1}$ and of butane via a very broad, almost unstructured absorption feature centered at $2950 \mathrm{~cm}^{-1}$ were both reported, along with ethane, $\mathrm{N}_{2} \mathrm{O}$, and other atmospheric trace gases. ${ }^{60}$ The quoted detection limits for methane and butane are 1-3 ppbv in atmospheric air using a procedure for recovering the spectrum of a broad absorber (butane) in the presence of one or more highly structured absorbers.

\section{Aerosol optical extinction}

By definition, an aerosol is a mixture of particles and a gas phase diluent, but typically the focus of aerosol studies is on the particles. Anthropogenically produced sulfate particles are important in global climate investigations, since they represent one of the few anthropogenic sources of negative radiative forcing. ${ }^{63-65}$ A great deal of attention has been focused recently on the impact of 'black carbon' particles (which produce warming of the atmosphere) on global climate change. ${ }^{63,64}$ The largest uncertainties in the global radiation budget are in the magnitude and direction of the radiative forcing by particles which absorb as well as scatter light. The measurement of optical extinction by CRD can be combined with separate measurements of scattering or absorption to derive all three key aerosol optical parameters: absorption, scattering, and extinction. Finally, optical extinction is directly related to visual range and clarity, an important quality-of-life issue.

In the CRD measurements of aerosols reported to date, the optical extinction (scattering plus absorption) of the aerosol has been measured at fixed wavelengths in the UV $(355 \mathrm{~nm})$, visible $(532,510,578 \mathrm{~nm})$ and near infrared $(1064 \mathrm{~nm}) .{ }^{18,19,66}$ All three instruments report detection limits of around $1 \mathrm{M} \mathrm{m}^{-1}$, a figure of merit used in atmospheric physics that corresponds to an extinction of 1 part per million per meter or $10^{-8} \mathrm{~cm}^{-1}$. There are also two as yet unreported systems which are currently being employed in field campaigns. ${ }^{67,68}$ One of these is a CW-CRD system especially designed for airborne sampling which contains an integral scattering measurement. ${ }^{68}$

By comparing ambient aerosols to a clean air background (produced by filtering the ambient air) ${ }^{19}$ Rayleigh scattering by air $\left(\mathrm{N}_{2}\right.$ and $\left.\mathrm{O}_{2}\right)$ is removed and only the particulate properties are measured. Another aspect of sample control that we have found particularly useful is the ability to size-selectively remove particles by using single stage impactors. Data from this type of experiment are shown in Fig. 4. The clean air baseline is very stable, making measurements of small extinctions possible. The whole air sample, with no pretreatment or filtering, shows much larger deviations than can originate from the measurement, due to the strong and rapid variation of large particulates which scatter light more efficiently than the more prevalent but uniform smaller ones. The first impactor, which eliminates most particles larger than $1.6 \mu \mathrm{m}$, seems to bear this out, since much of the variation is removed along with about $15 \%$ of the total extinction. The other size-cuts show essentially the same behavior, stable extinction at progressively lower levels. In principle, this information can be used to obtain an estimate of the particle density of each size cut, within the assumption of uniform particle refractive index.

Atmospheric particles are formed by a variety of physical and chemical processes and range in size, shape, refractive index, and chemical composition. This is one reason that the experiments reported to date monitor at more than one wavelength, since the wavelength dependence of the refractive index for different types of particles should provide an additional dimension of information. Since CRD measures total extinction, it is naturally complementary to nephelometry, a scattering measurement which is the most often applied bulk aerosol optical measurement and to PA spectroscopy which measures absorption. Quantitative checks of the accuracy of the CRD method are also possible through appropriate comparisons to these well-established techniques and are currently being pursued.

\section{Subject 2-indirect analysis methods incorporating CRD detection}

A group at DIAL (Diagnostic Instrumentation and Analysis Laboratory at Mississippi State University) has pioneered the use of cavity ring-down in tandem with traditional analytical preparation techniques. Using CRD absorption detection, they

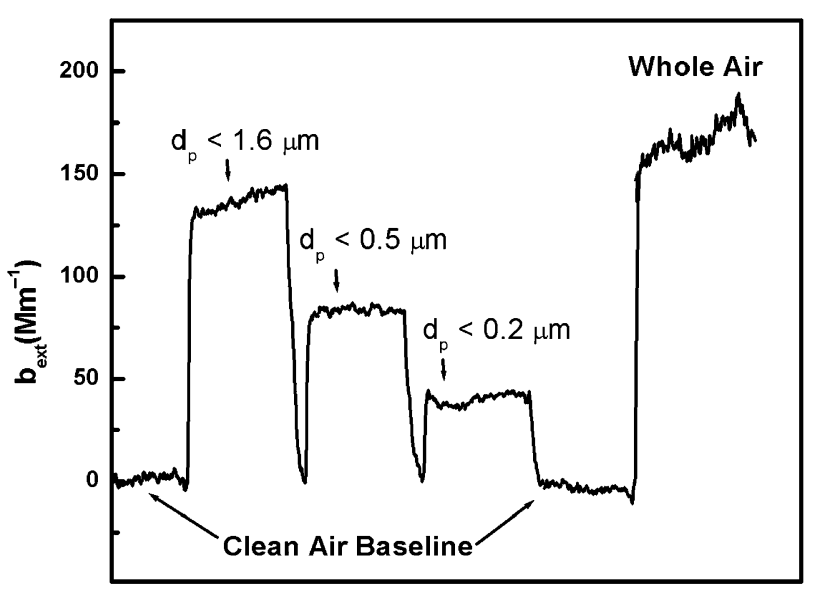

Fig. 4 Cavity ring-down transmissometer measurements of the visible $(532 \mathrm{~nm})$ optical extinction of ambient aerosol on a particularly hazy day. The deviations in the extinction observed with whole air are larger than those observed for clean air and are likely due to heterogeneously distributed large particles which are efficient scatterers. The size selected measurements are produced by using single stage impactors to remove a part of the ambient particle distribution. 
have demonstrated atomic analyses of mercury and lead using the inductively coupled plasma (ICP), electrothermal atomization (ETA) and batch chemical reaction (reduction) atomization methods. ${ }^{69-72}$ The success of these methods in extending the detection limit for atomic absorption spectroscopy to concentrations competitive with ICP-MS underscores the usefulness of the CRD approach.

Chemical reduction atomization of mercury with CRD detection $^{70}$ resulted in a detection limit of $27 \mathrm{pg} \mathrm{Hg}$, considerably lower than that reported for traditional AAS, and approaching that of the specialized Tekran detector (LOD $<1$ pg). Unfortunately this approach will likely be specific to mercury because of its volatility. The ETA method is more versatile; in principle any element could be analyzed using appropriate tunable lasers (and mirrors). An impressive detection limit for lead $(\sim 1 \mathrm{pg})$ is reported. ${ }^{72}$ Both the chemical reduction and ETA methods were limited by a slow time response for the pulsed CRD system used. These methods produce a transient burst of atoms with a width (FWHM) of a second or less. Considerable advances would be expected if either $\mathrm{kHz}$ pulsed lasers or $\mathrm{CW}-\mathrm{CRD}$ methods (which can sample at 100's of $\mathrm{Hz}$ ) were used to acquire the data, most likely with post-processing. An additional advantage of using the $\mathrm{CW}$ method would be the much smaller bandwidth of these systems, which will match better the narrow atomic linewidths.

The most generally useful system for routine CRD atomic analysis of environmental samples (where sample consumption is often not an issue) will probably be the ICP. Picogram detection limits for lead were also observed in the ICP-CRD experiment ${ }^{69}$ and considerable improvements may be possible by optimizing beam placement and discharge power. ${ }^{71}$ Since ICP is typically used with AES, production of excited states of the atoms is desirable, while in the CRD approach, only ground state atoms are typically probed, so cooler parts of the discharge are more effective. Probably the most exciting aspect of the ICP (and to a lesser extent the ETA) method is that a simple and inexpensive retrofit to existing instruments can be visualizedadding the CRD setup in an orthogonal axis to the existing AES setup will allow both detection methods to be used on a single torch.

\section{Subject 3-ancillary environmental studies using CRD}

A number of cavity ring-down studies which do not measure compounds in environmental samples are nonetheless interesting in that they help 'solve environmental problems.' The most obvious classes are measurements of the chemical kinetics and photo-physics of atmospherically important chemical reactions and compounds.

The investigation of chemical kinetics using CRD as a sensitive and time resolved detector of species density was originally demonstrated by Lin and co-workers. ${ }^{73}$ Since then, this group and others have used pulsed laser photolysis with pulsed-CRD detection of radicals to measure a host of atmospherically relevant reaction rate parameters (rate coefficients and their dependence on temperature and pressure). ${ }^{22,74-76}$ There have also been measurements of the photodissociation dynamics of molecules using similar methods. ${ }^{77-81}$ These photo-physical reactions are known to be important in atmospheric chemistry and physics. Incomplete knowledge of key reaction rates and product yields continue to account for much of the uncertainty surrounding atmospheric chemical models.

The most straightforward applications of CRD in photophysical studies are the accurate measurements of absolute absorption strengths of atmospheric molecules. ${ }^{82-86}$ One aspect of this work is measuring overtone spectra of molecules which are too weak to be studied by normal absorption spectroscopy, but which are of known importance to the global radiation budget. Often these spectra are quite broad, making them difficult to measure with other sensitive absorption methods which use rapid frequency modulation. For species exhibiting broad spectra (e.g., $\mathrm{HNO}_{3}$ and $\mathrm{H}_{2} \mathrm{O}_{2}$ ) the cavity ring-down measurements are the best to date. ${ }^{86}$

An example of kinetic measurements by CRD is our group's recent report on the reaction kinetics of peroxy radicals. ${ }^{87}$ These radicals, generically denoted $\mathrm{RO}_{2}$, are formed in the atmosphere from the addition of molecular oxygen to the highly unstable radicals which result from the reactions of hydroxyl radical with volatile organic compounds. ${ }^{88-90}$ They are known to be key intermediates in tropospheric ozone formation through their reaction with NO. ${ }^{91,92}$ Reactions between different peroxy radicals, which may play important roles in the remote troposphere ${ }^{93}$ remain poorly characterized because of poor selectivity in their UV absorption detection. ${ }^{94}$

Our method uses pulsed laser photolytic production of radicals and near-infrared CW-CRD $(\sim 1300 \mathrm{~nm})$ to achieve sensitive and selective detection. ${ }^{87}$ The spectra of the ethyl peroxy and methyl peroxy radicals ${ }^{95}$ has been measured by pulsed CRD recently, along with that of $\mathrm{CF}_{3} \mathrm{O}_{2}{ }^{96}$ and our measured spectra (Fig. 5) agree well with those reported previously. By monitoring the absorption of ethyl peroxy radicals near $1316.4 \mathrm{~nm}$ and of methyl peroxy radical near $1335.1 \mathrm{~nm}$, it is possible to monitor the self-reactions of the
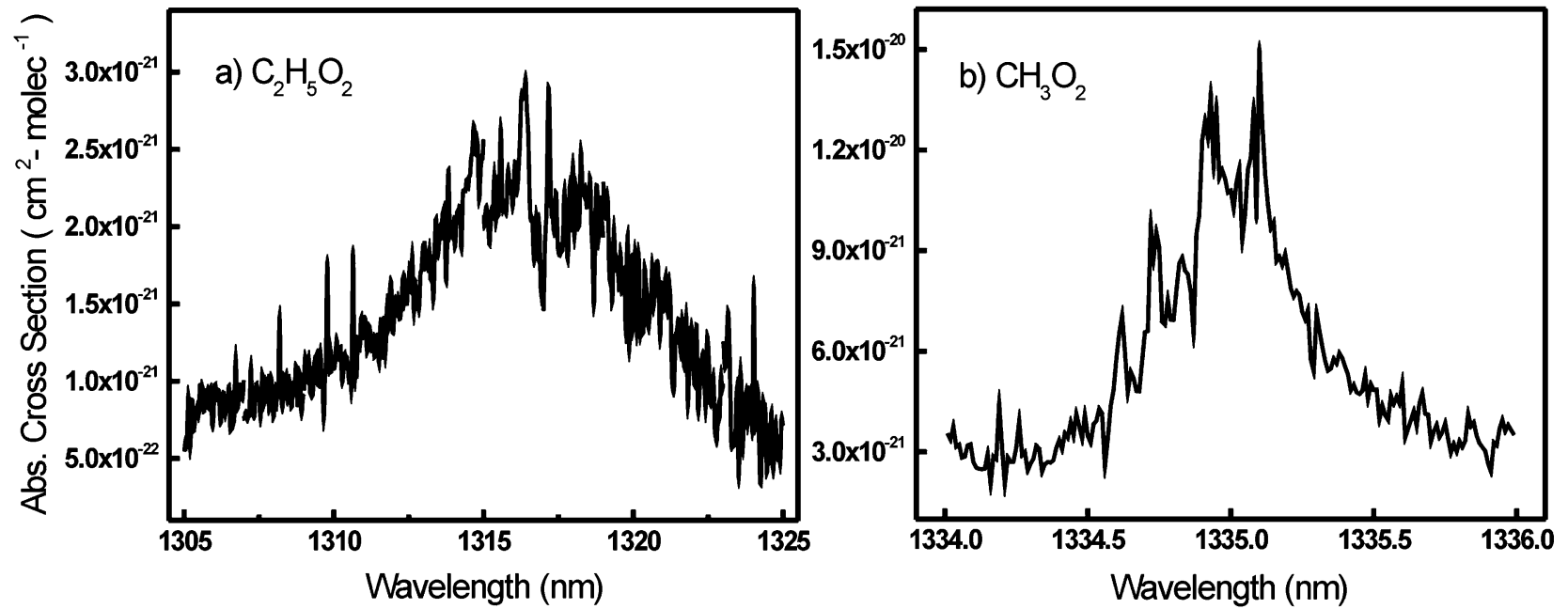

Fig. 5 The spectra of the peroxy radicals $\mathrm{C}_{2} \mathrm{H}_{5} \mathrm{O}_{2}$ (a) and $\mathrm{CH}_{3} \mathrm{O}_{2}$ (b) measured in our pulsed laser photolysis/CW-CRD reactor system. The radicals may be produced alone or together by $193 \mathrm{~nm}$ photolysis of one or both precursors, acetone (for $\mathrm{CH}_{3} \mathrm{O}_{2}$ ) and 3-pentanone $\left(\right.$ for $\mathrm{C}_{2} \mathrm{H}_{5} \mathrm{O}_{2}$ ). Reproduced with permission from J. Phys. Chem. A, 2002, 106, p. 8891. Copyright 2002 American Chemical Society. 
radicals or the cross-reaction between the radicals by observing the decay of the individual radical signal (Fig. 6). Although our instrument is limited to the near-infrared and is mainly intended for the study of the peroxy radical cross-reactions, we feel that the principle should be generally useful to the study of freeradical kinetics as was the seminal work of $\mathrm{Yu}$ and Lin. ${ }^{73}$

\section{Future outlook for CRD in solving environmental problems}

From the various studies presented in this review, it is clear that the particular strengths of cavity ring-down (i.e., sensitivity, ready quantitation, generality, simple and robust operation, good time resolution) make it useful in environmental applications, particularly direct gas-phase monitoring. It should also be stressed that CRD suffers from many of the same limitations as normal absorption spectroscopy. Total extinction (and only total extinction) is measured, meaning that anything in the sample matrix that absorbs or scatters the light is included. This can cause interference problems and can also result in a degradation of the predicted sensitivity enhancement. An excellent example is the large deviations observed in the $\mathrm{NO}_{3}$ signal of ref. 42 when the contribution of aerosol particleinduced scattering is not removed by filtering. There are many other examples of this problem, ranging from small degradations of the signal to noise ratio of the measurement due to an unsteady extinction contributor, to the complete interference observed in the measurement of $\mathrm{NO}$ in the presence of $\mathrm{CO}_{2}$ shown in Fig. 7 of ref. 50. Usually the best way to circumvent these problems is to move to another spectral region which is freer from interference. Unlike other popular optical experiments (e.g., fluorescence, REMPI, photo-acoustic spectroscopy, etc.), CRD is not a zero-background measurement, so increasing the exciting laser intensity and/or the gain of the detector does not generally yield a corresponding improvement in sensitivity. Beyond optimizing the experimental design (i.e., mirror reflectivity, alignment for single mode excitation) the only way to improve the signal to noise of the optical extinction measurement is to increase the signal averaging. ${ }^{5}$

The use of CRD in atmospheric field monitoring will probably continue to expand, especially as good mid-infrared laser sources become cheaper and more readily available. This

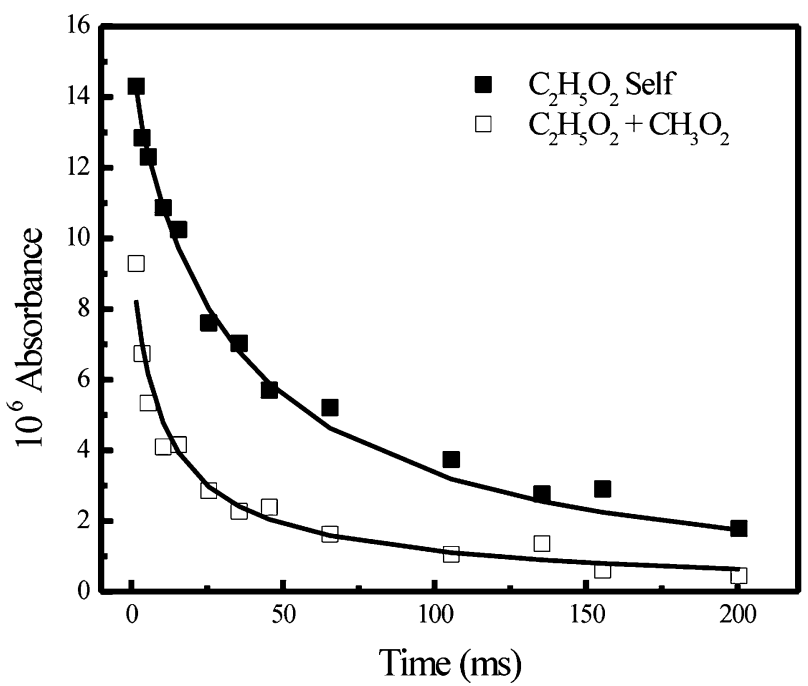

Fig. 6 Sample kinetic decays for ethyl peroxy radical $\left(\mathrm{C}_{2} \mathrm{H}_{5} \mathrm{O}_{2}\right)$ monitored on the strong bandhead near $1316 \mathrm{~nm}$. The filled squares are the observed decay when only ethyl peroxy is present, while the open squares are the much more rapid decay when an equal quantity of the more reactive methyl peroxy radical $\left(\mathrm{CH}_{3} \mathrm{O}_{2}\right)$ is also present. Least squares fitting of an ensemble of selected experiments will allow extraction of the elementary crossreaction rate coefficient. Reproduced with permission from J. Phys. Chem. A, 2002, 106, p. 8891. Copyright 2002 American Chemical Society. region of the spectrum provides access to a large number of molecules and careful selection of monitoring wavelengths can help mitigate interference effects which are prevalent here. The use of multiple wavelength measurements will likely be useful in avoiding interference problems. ${ }^{33,34,36}$ Other 'relatives' of CRD (e.g., CEAS $)^{6-8}$ may also figure prominently, since they are often less experimentally demanding. For those species that possess distinct absorption features in the visible and near-UV, those wavelengths typically offer less interference. If a method is purported to be 'sufficiently sensitive' for atmospheric monitoring, the claims should be based on actual measurement, not noise equivalent absorption, within a sample matrix that is similar to the atmosphere. (Failing that, a reasonable estimate of the possible sources and sizes of interferences should be discussed.) Optimally, measurements of atmospheric levels of the target compound should be presented and compared with the results obtained with more standard measurement methods, if any exist.

The use of CRD as the detector in atomic spectroscopy has only begun to be explored but the results are very promising. ${ }^{69-72}$ The use of CRD in tandem with more conventional analytical separation and sample preparation techniques may become more common. At the time of this review, the use of CRD as a detector in chromatography has not been demonstrated. An obvious example would be to use the newly reported liquid-phase CRD methods ${ }^{97}$ as the detector in liquid chromatography. These methods (chromatography and atomic spectroscopy) are the most likely avenues for CRD to make an impact on environmental work in the condensed phases (solid and liquid).

The discussion of the ancillary CRD-related topics of interest to environmental chemistry was necessarily brief. A large number of studies which are less obviously related to environmental chemistry could have been included. The quantitative nature of CRD is often exploited to produce much more reliable measurements of species concentrations and photo-physical properties than can otherwise be obtained.

While the number of papers published citing cavity ringdown measurement as a title (or keyword) level subject will probably peak soon, the usefulness of the method has likely only begun to be explored. This is especially true in environmental analytical chemistry, where methods are typically only widely applied after their merit is proven. As trace level analysis continues to become more important and as the demands to reliably quantify low level contaminants increases, the advantages of CRD will make it another of the standard components in the analytical chemist's toolbox.

\section{References}

1 A. O'Keefe and O. Lee, Am. Lab. (Shelton, Conn.), 1989, 21, 19.

2 G. Berden, R. Peeters and G. Meijer, Int. Rev. Phys. Chem., 2000, 19, 565-608.

3 Cavity-Ringdown Spectroscopy An Ultratrace-Absorption Measurement Technique, ed. K. W. Busch and M. A. Busch, American Chemical Society, distributed by Oxford University Press, Washington, DC, 1999.

4 J. J. Scherer, J. B. Paul and R. J. Saykally, Chem. Rev., 1997, 97, 25.

5 M. D. Wheeler, S. M. Newman, A. J. Orr-Ewing and M. N. R. Ashfold, J. Chem. Soc., Faraday Trans., 1998, 94, 337-351.

6 A. O'Keefe, J. J. Scherer and J. B. Paul, Chem. Phys. Lett., 1999, 307, 343.

7 A. O'Keefe, Chem. Phys. Lett., 1998, 293, 331-336.

8 R. Engeln, G. Berden, R. Peeters and G. Meijer, Rev. Sci. Instrum., 1998, 69, 3763-3769.

9 J. Ye, L.-S. Ma and J. L. Hall, ACS Symp. Ser., 1999, 720, 233-253.

10 P. Zalicki and R. N. Zare, J. Chem. Phys., 1995, 102, 2708.

11 K. K. Lehmann and D. Romanini, J. Chem. Phys., 1996, 105, 10263. 
12 J. T. Hodges, J. P. Looney and R. D. van Zee, J. Chem. Phys., 1996, 105, 10278-10288

13 J. B. Paul and R. J. Saykally, Anal. Chem., 1997, 69, 287A.

14 A. E. Siegman, Lasers, University Science Books, Mill Valley, CA, 1986.

15 T. G. Spence, C. C. Harb, B. A. Paldus, R. N. Zare, B. Willke and R. L. Byer, Rev. Sci. Instrum., 2000, 71, 347-353.

16 D. Romanini and K. K. Lehmann, J. Chem. Phys., 1995, 102, 633-642.

17 A. O'Keefe and D. A. G. Deacon, Rev. Sci. Instrum., 1988, 59, 2544-2551.

18 J. E. Thompson, B. W. Smith and J. D. Winefordner, Anal. Chem., 2002, 74, 1962-1967.

19 J. D. Smith and D. B. Atkinson, Analyst, 2001, 126, 1216-1220.

20 R. Engeln, G. Berden, E. van den Berg and G. Meijer, J. Chem. Phys., 1997, 107, 4458-4467.

21 American Chemical Society, Anal. Chem., 1990, 62, 91.

22 D. B. Atkinson and J. W. Hudgens, J. Phys. Chem. A, 1997, 101, 3901-3909.

23 J. P. Looney, J. T. Hodges and R. D. Van Zee, ACS Symp. Ser., 1999, 720, 93-105.

24 M. Muertz, B. Frech and W. Urban, Appl. Phys. B: Lasers Opt., 1999, B68, 243-249.

25 K. W. Busch, A. Hennequin and M. A. Busch, ACS Symp. Ser., 1999, 720, 34-48.

26 D. Romanini, A. A. Kachanov, N. Sadeghi and F. Stoeckel, Chem. Phys. Lett., 1997, 264, 316-322.

27 K. J. Schulz and W. R. Simpson, Chem. Phys. Lett., 1998, 297, 523.

28 B. A. Paldus, PhD Thesis, Stanford University, 1998.

29 B. A. Paldus, J. S. Harris, Jr, J. Martin, J. Xie and R. N. Zare, J. Appl. Phys., 1997, 82, 3199-3204.

30 D. Romanini, A. A. Kachanov and F. Stoeckel, Chem. Phys. Lett. 1997, 270, 538.

31 B. A. Paldus, C. C. Harb, T. G. Spence, B. Wilke, J. Xie, J. S. Harris and R. N. Zare, J. Appl. Phys., 1998, 83, 3991-3997.

32 K. K. Lehmann, U.S. Pat. applied for, Princeton University, USA.

33 J. J. Scherer, Chem. Phys. Lett., 1998, 292, 143.

34 J. J. Scherer, J. B. Paul, H. Jiao and A. O'Keefe, Appl. Opt., 2001, 40, 6725-6732.

35 A. Czyzewski, S. Chudzynski, K. Ernst, G. Karasinski, L. S. Kilianek, A. Pietruczuk, W. Skubiszak, T. Stacewicz, K. Stelmaszczyk, B. Koch and P. Rairoux, Opt. Commun., 2001, 191, 271-275.

36 S. M. Ball, I. M. Povey, E. G. Norton and R. L. Jones, Chem. Phys. Lett., 2001, 342, 113-120.

37 L. Pillier, C. Moreau, X. Mercier, J. F. Pauwels and P. Desgroux, Appl. Phys. B: Lasers Opt., 2002, 74, 427-434.

38 W. P. Arnott, H. Moosmuller and D. F. Rogers, Atmos. Environ., 1999, 33, 2845-2842.

39 B. J. Finlayson-Pitts and J. N. Pitts, Chemistry of the Upper and Lower Atmosphere, Academic Press, San Diego, CA, 2000.

40 D. A. Skoog, F. J. Holler and T. A. Niemann, Principles of Instrumental Analysis, Saunders College Publishing, Philadelphia, PA, 5th edn., 1998

41 W. P. Arnott, H. Moosmuller and J. W. Walker, Rev. Sci. Instrum., 2000, 71, 4545-4552.

42 S. S. Brown, H. Stark, S. J. Ciciora and A. R. Ravishankara, Geophys. Res. Lett., 2001, 28, 3227-3230.

43 J. H. Seinfeld and S. N. Pandis, Atmospheric Chemistry and Physics: From Air Pollution to Climate Change, John Wiley \& Sons, Inc. New York, NY, 1998.

44 R. Evertsen, A. Staicu, N. Dam, A. Van Vliet and J. J. Ter Meulen, Appl. Phys. B: Lasers Opt., 2002, 74, 465-468.

45 J. Lauterbach, D. Kleine, K. Kleinermanns and P. Hering, Appl. Phys. B: Lasers Opt., 2001, 72, 631.

46 P. Dupre, C. R. Acad. Sci., Ser. IV: Phys., Astrophys., 2001, 2 , 929-964.

47 D. Romanini, P. Dupre and R. Jost, Vib. Spectrosc., 1999, 19, 93.

48 A. Staicu, R. Evertsen, N. J. Dam and J. J. ter Meulen, Proc. SPIEInt. Soc. Opt. Eng., 2001, 4430, 620-627.

49 R. Vasudev, A. Usachev and W. R. Dunsford, Environ. Sci. Technol., 1999, 33, 1936.

50 A. A. Kosterev, A. L. Malinovsky, F. K. Tittel, C. Gmachl, F. Capasso, D. L. Sivco, J. N. Baillargeon, A. L. Hutchinson and A. Y. Cho, Appl. Opt., 2001, 40, 5522-5529.

51 M. D. King, E. M. Dick and W. R. Simpson, Atmos. Environ., 2000, 34, 685-688.

52 S. S. Brown, personal communication, 2002.

53 L. Wang and J. Zhang, Environ. Sci. Technol., 2000, 34, $4221-4227$.
54 G. W. Harris, W. P. Carter and A. M. Winer, Jr, Environ. Sci. Technol., 1982, 16, 414.

55 R. T. Jongma, M. G. H. Boogaarts, I. Holleman and G. Meijer, Rev Sci. Instrum., 1995, 66, 2821-2827.

56 S. Spuler, M. Linne, A. Sappey and S. Snyder, Appl. Opt., 2000, 39, 2480-2486.

57 H. R. Barry, L. Corner, G. Hancock, R. Peverall and G. A. D. Ritchie, Chem. Phys. Lett., 2001, 333, 285-289.

58 C. Ishibashi and H. Sasada, Jpn. J. Appl. Phys., Part 1, 1999, 38, 920-922.

59 D. Kleine, M. Murtz, J. Lauterbach, H. Dahnke, W. Urban, P. Hering and K. Kleinermanns, Isr. J. Chem., 2001, 41, 111-116.

60 K. W. Aniolek, T. J. Kulp, B. A. Richman, S. E. Bisson, P. E. Powers and R. L. Schmitt, Proc. SPIE-Int. Soc. Opt. Eng., 1999, 3758, 62-73.

61 M. Muertz, T. Kayser, D. Kleine, S. Stry, P. Hering and W. Urban, Proc. SPIE-Int. Soc. Opt. Eng., 1999, 3758, 53-61.

62 H. Dahnke, D. Kleine, W. Urban, P. Hering and M. Murtz, Appl. Phys. B: Lasers Opt., 2001, 72, 121-125.

63 J. Haywood and O. Boucher, Rev. Geophys., 2000, 38, 513-543.

64 J. T. Houghton, Y. Ding, D. J. Griggs, M. Noguer, P. J. van der Linden, X. Da, K. Maskell and C. A. Johnson, Cambridge University Press, Cambridge, 2001.

65 G. Myhre, F. Stordal, K. Restad and I. S. A. Isaksen, Tellus, Ser. B:, 1998, 50B, 463-477.

66 A. D. Sappey, E. S. Hill, T. Settersten and M. A. Linne, Opt. Lett., 1998, 23, 954-956.

67 H. Moosmuller, personal communication, 2002

68 A. W. Strawa, R. Castaneda, T. Owano, D. S. Baer and B. A. Paldus, personal communication, 2002.

69 G. P. Miller and C. B. Winstead, J. Anal. At. Spectrom., 1997, 12, 907.

70 S. Tao, F. J. Mazzotti, C. B. Winstead and G. P. Miller, Analyst, 2000, 125, 1021-1023

71 C. Wang, F. J. Mazzotti, G. P. Miller and C. B. Winstead, Appl. Spectrosc., 2002, 56, 386-397.

72 C. B. Winstead, F. J. Mazzotti, J. Mierzwa and G. P. Miller, Anal. Commun., 1999, 36, 277-279.

73 T. Yu and M. C. Lin, J. Am. Chem. Soc., 1993, 115, 4371-4372.

74 D. B. Atkinson, J. W. Hudgens and A. J. Orr-Ewing, J. Phys. Chem. A, 1999, 103, 6173-6180.

75 T. Yu and M. C. Lin, J. Am. Chem. Soc., 1994, 116, 9571-9576.

76 T. Yu and M. C. Lin, J. Phys. Chem., 1994, 98, 2105-2109.

77 L. Zhu and G. Johnston, J. Phys. Chem., 1995, 99, 15114-15119.

78 L. Zhu and D. Kellis, Chem. Phys. Lett., 1997, 278, 41-48.

79 L. Zhu, D. Kellis and C. F. Ding, Book of Abstracts, 214th ACS National Meeting, Las Vegas, NV, September 7-11, 1997, COLL120.

80 L. Zhu and C.-F. Ding, Chem. Phys. Lett., 1997, 265, 177-184.

81 Y. Chen, W. Wang and L. Zhu, J. Phys. Chem. A, 2000, 104, 11126-11131.

82 D. L. Huestis, R. A. Copeland, K. Knutsen, T. G. Slanger, R. T. Jongma, M. G. H. Boogaarts and G. Meijer, Can. J. Phys., 1994, 72 1109-1121.

83 S. Xu, D. Dai, J. Xie, G. Sha and C. Zhang, Chem. Phys. Lett., 1999 , 303, 171-175

84 J. P. Looney, R. D. Van Zee and J. T. Hodges, AIP Conference Proceedings, 1999, 467, 275-285.

85 D. Kleine, S. Stry, J. Lauterbach, K. Kleinermanns and P. Hering, Chem. Phys. Lett., 1999, 312, 185-190.

86 S. S. Brown, R. W. Wilson and A. R. Ravishankara, J. Phys. Chem. A, 2000, 104, 4976.

87 D. B. Atkinson and J. L. Spillman, J. Phys. Chem. A, 2002, 106, 8891.

88 R. Atkinson, Atmos. Environ., 1990, 24A, 1-41.

89 R. Atkinson, J. Phys. Chem. Ref. Data, 1997, 26, 215-290.

90 R. Atkinson and J. Arey, Acc. Chem. Res., 1998, 31, 574-583.

91 R. G. Derwent, M. E. Jenkin, S. M. Saunders and M. J. Pilling, Atmos. Environ., 1998, 32, 2429-2441.

92 W. P. L. Carter, J. Air Waste Manage. Assoc., 1994, 44, 881-899.

93 M. E. Jenkin, S. M. Saunders and M. J. Pilling, Atmos. Environ., 1997, 31, 81-104.

94 E. Villenave and R. Lesclaux, J. Phys. Chem., 1996, 100, 14372-14382.

95 M. B. Pushkarsky, S. J. Zalyubovsky and T. A. Miller, J. Chem. Phys., 2000, 112, 10695-10698.

96 S. J. Zalyubovsky, D. Wang and T. A. Miller, Chem. Phys. Lett., 2001, 335, 298-304.

97 A. J. Hallock, E. S. F. Berman and R. N. Zare, Anal. Chem., 2002, 74, 1741-1743. 